\title{
VERITAS Observations of 1ES 1218+304 during the 2019 VHE High State
}

\section{Stephan O'Brien*广}

Department of Physics, McGill University, Montreal, Canada

E-mail: stephan.obrien@mcgill.ca

1ES $1218+304$ is a moderate-redshift $(\mathrm{z}=0.182)$ high-frequency-peaked BL Lac object $(\mathrm{HBL})$. Detected by both the MAGIC and VERITAS gamma-ray observatories, 1ES 1218+304 is frequently monitored by VERITAS as part of its long-term blazar monitoring program. On the 3rd of January 2019, during a regularly scheduled blazar snapshot, VERITAS observed 1ES 1218+304 to have an elevated VHE flux state which continued through January 5 (ATEL \#12360). In addition to VERITAS observations, MAGIC also detected elevated VHE emission (ATEL \#12354) and Swift-BAT/XRT and Tuorla observations, in X-ray and optical respectively, showed increased multiwavelength activity at the time of the VERITAS observations. In this work, VERITAS observations of 1ES 1218+304 taken as part of a target of opportunity campaign during this elevated state, are presented.

36th International Cosmic Ray Conference -ICRC2019-

July 24th - August 1st, 2019

Madison, WI, U.S.A.

\footnotetext{
* Speaker.

${ }^{\dagger}$ For the VERITAS Collaboration
} 


\section{Introduction}

1ES $1218+304$ is a high-frequency-peaked BL Lac Object located at a redshift of $\mathrm{z}=0.182$. It was discovered as a VHE emitter by the MAGIC collaboration [1] during an observation campaign in 2005. During this campaign no significant flux variability was observed on the time-scales of days. VERITAS has also detected 1ES 1218+304, and during a 2008-2009 observation campaign it was observed to have a mean flux of 7\% Crab [2]. Between the nights of 25th January 2009 and 5th February 2009, day-scale variability was observed with the flux reaching a peak of $\sim 20 \% \mathrm{Crab}$ on the night of 30th January 2009.

During late 2018, 1ES 1218+304 entered an active state across multiple wavelengths, with enhanced activity at both X-ray ${ }^{1}$ and optical energies ${ }^{3}$. During a regularly scheduled monitoring observation on 3rd January 2019, VERITAS observed an enhanced TeV level of $>20 \%$ from $1 \mathrm{ES} 1218+304$ [3]. In response to this elevated state, target of opportunity (ToO) observations were taken the following night by VERITAS. MAGIC also reported an elevated state during this period [4]. VERITAS continued to monitor 1ES 1218+304 at a high-cadence until 3rd April 2019. During this period a historic peak in the X-ray count rate was observed by Swift-XRT [5].

In these proceedings, the gamma-ray data taken by VERITAS and Fermi-LAT from the 20182019 season are presented. In Section 2 the observations taken by VERITAS and the FermiLAT data are described. In Section 3 the gamma-ray light curves of 1ES 1218+304 are discussed and a search for flux variability is performed. In Section 4 the gamma-ray spectral energy distribution (SED) for the time-averaged data set is obtained. In addition the SED for an exceptional nightly scale flare is examined. Finally in Section 5, the results are summarized.

\section{Observations}

VERITAS (Very Energetic Radiation Imaging Telescope Array System) is a gound-based gamma-ray detector, sensitive to gamma rays in the $100 \mathrm{GeV}->30 \mathrm{TeV}$ energy range. Located at the Fred Lawrence Whipple Observatory (FLWO) in southern Arizona USA (31 40N, 110 57W, $1.3 \mathrm{~km}$ a.s.1.), the VERITAS array consists of four 12-m imaging atmospheric-Cherenkov telescopes. Each telescope has a Davies-Cotton-design segmented mirror dish with 345 facets. At the focal plane of each dish, lies a a 499 PMT camera, which has a total field of view of $3.5^{\circ}$. The $68 \%$ containment radius for a $1 \mathrm{TeV}$ photon is $<0.1^{\circ}$, and the pointing accuracy is $<50^{\prime \prime}$. In its current configuration VERITAS can detect a source with flux $1 \%$ that of the Crab Nebula in $\sim 25$ hours of observations and has an energy resolution of $15-25 \%$. For full details of VERITAS and its performance see [6].

VERITAS data taken between 58461 and 58600 MJD (12th December 2018 and 27th April 2019) were analyzed using the two standard VERITAS analysis packages and excellent agreement was found. Boosted decision tree optimized a priori for soft-spectrum point-source analysis, were used in gamma/hadron separation [7]. In total the data presented here corresponds to 12.2 hours of dead-time-corrected exposure. This results in the detection of 556.54 excess gamma-ray-like

\footnotetext{
${ }^{1}$ Swift-BAT - https://swift.gsfc.nasa.gov/results/transients/weak/QSOB1218p304/

${ }^{2}$ Swift-XRT - https://www.swift.psu.edu/monitoring/source.php?source=1ES1218+304

${ }^{3}$ Tuorla - http://users.utu.fi/kani/1m/1ES_1218+304.html
} 
events (1087 ON-source, $3789 \mathrm{OFF}$-source, mean On/Off normalization of 0.14), giving a detection of 19.4 standard deviations above the background $(\sigma)$ [8]. The correlated significance sky map of the region is shown in Figure 1, where 1ES 1218+304 is clearly visible at the centre. In performing background estimation, $0.35^{\circ}$ radius circles around bright stars are excluded from background estimation. In addition, there is a known near-by VHE emitter, $1 \mathrm{ES} 1215+303^{4}$, located within the region. A $0.35^{\circ}$ region around the location of 1 ES $1215+303$ is also excluded. The exclusion regions are shown in Figure 1 as magenta circles.

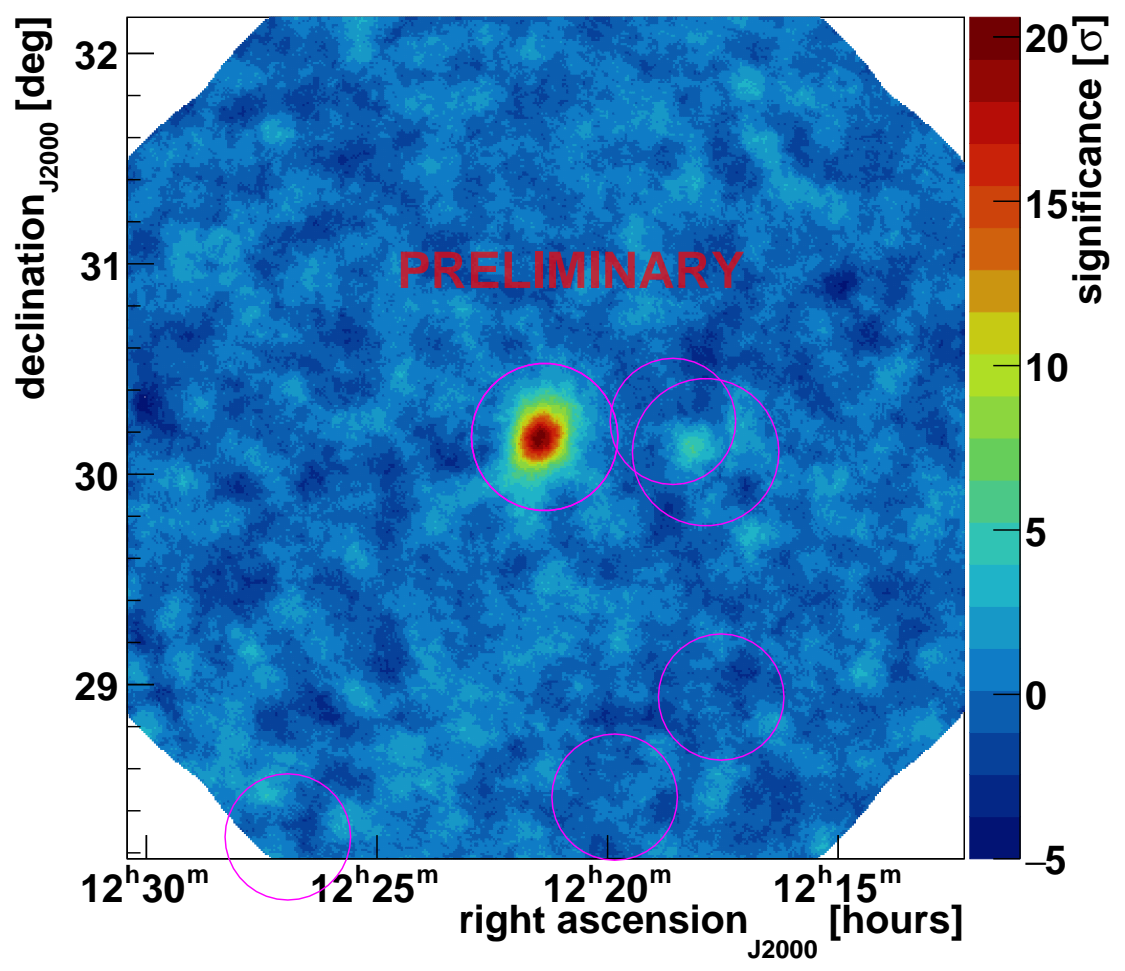

Figure 1: Correlated significance sky map for the region of interest. The magenta circles indicate regions excluded from the background estimation.

The large area telescope on board the Fermi space-based observatory (Fermi-LAT [9]) is a pairconversion telescope sensitive to gamma rays in the $\sim 20 \mathrm{MeV}->300 \mathrm{GeV}$ energy range. The large FoV of Fermi-LAT allows for a full survey of the sky every 3 hours. Data taken by FermiLAT was analyzed using the Fermipy python package [10], using Science Tools ${ }^{5}$ (Fermitools $\mathrm{v} 1.0 .0)$. "Source" class events (evclass=128) from both the front and back (evtype=3), with energies between $100 \mathrm{MeV}$ and $300 \mathrm{GeV}$ were considered. Only photons originating from within a $15^{\circ}$ radius of 1 ES $1218+304$ were considered and a zenith angle cut of $90^{\circ}$ was applied to avoid contamination from the Earth's limb. A binned-likelihood analysis was performed, where the 3FGL catalog [11] details of nearby sources were included in the model. The fit was performed with the

\footnotetext{
${ }^{4}$ http://tevcat.uchicago.edu/?mode $=1 ; \mathrm{id}=219$

${ }^{5}$ https://fermi.gsfc.nasa.gov/ssc/data/analysis/documentation/Cicerone/
} 
spectral normalization of sources within $3^{\circ}$ of $1 \mathrm{ES} 1218+304$ allowed to vary and the rest frozen to their 3FGL average. The normalization of the isotropic and galactic diffuse components were also fit as free parameters. 1ES $1218+304$ is significantly detected by Fermi-LAT during this period, with a TS of $441(\sqrt{\mathrm{TS}} \sim \sigma)$, assuming a power-law model.

\section{Flux Analysis}

The nightly-binned flux $(E>150 \mathrm{GeV})$ obsevered by VERITAS was obtained assuming a power-law model with the spectral index frozen to that of the time-averaged best-fit value of $\Gamma=$ -3.25 (see Section 4 and Table 1). The VERITAS flux is shown in the top panel of Figure 2. 95\% confidence level (C.L.) upper limits were obtained for bins with less than $2 \sigma$ excess significance or $<5$ ON-source events. The time-averaged flux during this period was determined to be $F(E>$ $150 \mathrm{GeV})=(3.88 \pm 0.26) \times 10^{-11} \mathrm{~cm}^{-2} \mathrm{~s}^{-1}$ or $11 \% \mathrm{Crab}$. To detect significant change points in the VERITAS light curves, a Bayesian-block analysis [12] was performed using the astropy python package [13]. In performing this analysis, a false-alarm probability of 0.01 was chosen. To avoid biasing the results, all data points, regardless of their significance, were included in the analysis. The time-binned fluxes are shown as green squares in the top panel of Figure 2. The Bayesian-block algorithm picks out a significant day-scale bin on the night of $58490 \mathrm{MJD}$. On this night, the flux of 1ES $1218+304$ reaches $F(E>150 \mathrm{GeV})=(8.44 \pm 0.72) \times 10^{-11} \mathrm{~cm}^{-2} \mathrm{~s}^{-1}$, or $23 \%$ Crab. This is $\sim 2$ times higher than the time-averaged flux of the campaign. The spectral analysis of data taken on this night is presented in Section 4.

Weekly-binned Fermi-LAT light curves are obtained in the $E>100 \mathrm{MeV}$ and $E>1 \mathrm{GeV}$ energy ranges. In obtaining the light curve, the fit parameters for other sources within the FoV are frozen to their best-fit values. The flux $E>100 \mathrm{MeV}$, and $E>1 \mathrm{GeV}$ are shown in the bottom and middle panels of Figure 2. During the campaign, the time-averaged flux in the $E>100 \mathrm{MeV}$, and $E>1 \mathrm{GeV}$ energy ranges were determined to be $(3.55 \pm 0.53) \times 10^{-8} \mathrm{~cm}^{-2} \mathrm{~s}^{-1}$ and $(6.43 \pm$ $0.64) \times 10^{-9} \mathrm{~cm}^{-2} \mathrm{~s}^{-1}$, respectively, and are shown as dashed black lines in the bottom and middle panels. One notices that the flux above $1 \mathrm{GeV}$ reaches a local maximum which is temporally consistent with the significant day-scale block picked out by the Bayesian-block algorithm. While the $E>1 \mathrm{GeV}$ flux shows moderate elevation around $58490 \mathrm{MJD}$, the $E>100 \mathrm{MeV}$ flux shows a level consistent with the campaign mean. This could be an indication of higher-energy variability, particularly about the inverse-Compton peak (IC-peak) of the SED. To examine this behaviour, the SED is examined in the next section.

\section{Spectral Analysis}

The VERTIAS spectral analysis was performed using a forward-folding binned-likelihood method, originally developed for the CAT experiment [14]. This method forward-folds the instrument response function with the spectral model to obtain a prediction of the model observed counts, hence allowing for the Poisson based likelihood maximization to be performed. The timeaveraged spectrum was obtained by considering all good-weather data. The data were divided into bins spaced 0.15 in log-space, and the best-fit power-law model was obtained. In applying the fit, all bins up to and including the first insignificant bin $(<2 \sigma$ excess), were included in the fit. This 

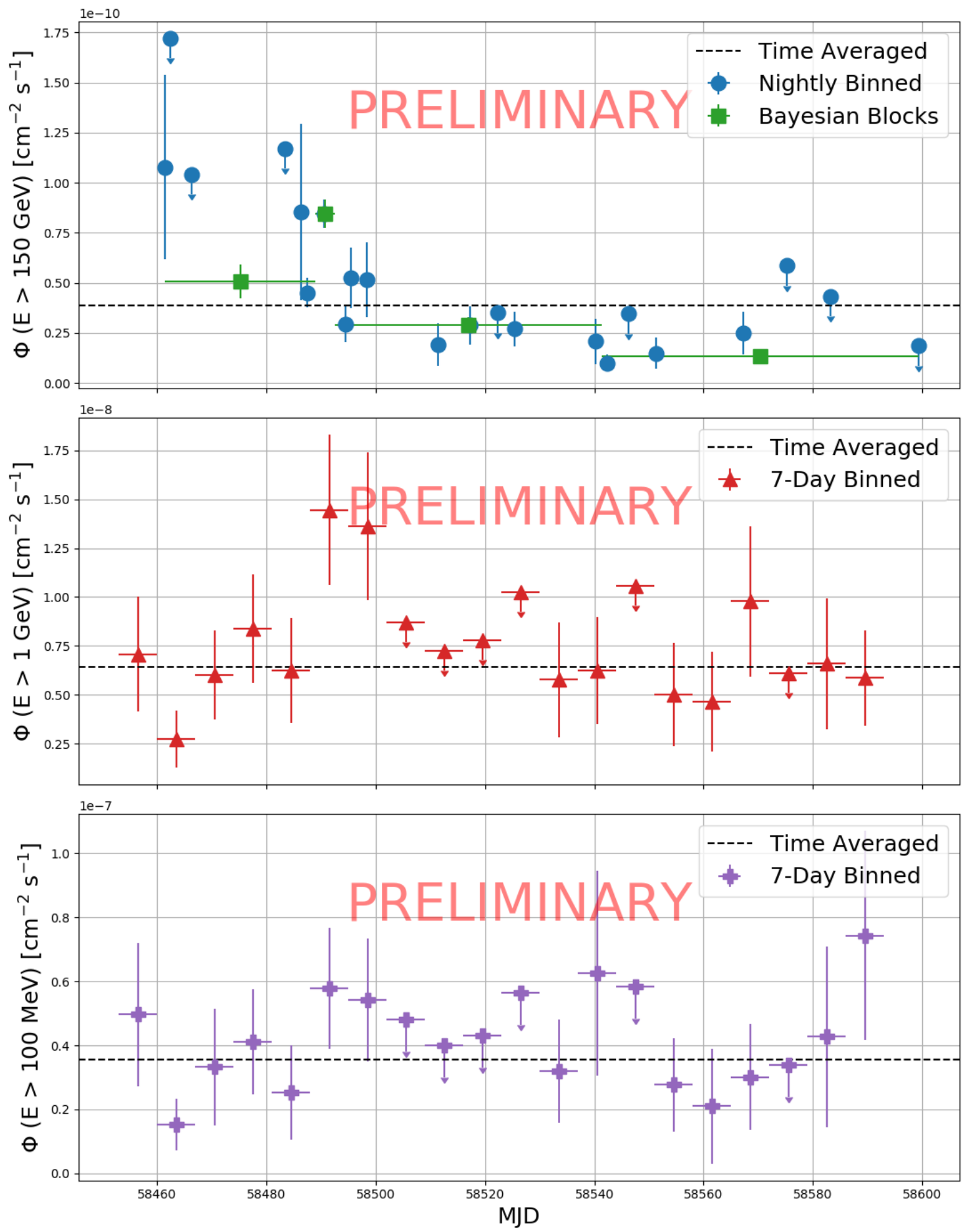

Figure 2: Gamma-ray light curve for 1ES 1218+304 for the period considered in this analysis. The top panel shows the VERITAS flux $>150 \mathrm{GeV}$, with the blue circles denoting the nightly-binned flux and the green squares denoting the average flux across the block period. The middle panel shows the Fermi-LAT flux $>1 \mathrm{GeV}$. The bottom panel shows the Fermi-LAT flux $>100 \mathrm{MeV}$. See text for more details. 
aims to the reduce the effect of biasing ones data towards positive fluctuations. Spectral points are obtained by freezing the spectral shape parameters (i.e. spectral index) and fitting the model across each energy bin, allowing the normalization to vary. 95\% C.L. upper limits are obtained by examining the profile likelihoods of insignificant energy bins, and obtained using a similar method as described by [15]. The details of best-fit time-averaged and "flare"-night (58490 MJD) are shown in Table 1 and are plotted as green ' $X$ 's and purple diamonds, respectively, in Figure 3. Note the points in Figure 3 are deabsorbed for EBL attenuation assuming a [16] EBL model. The flare-night SED shows a $\sim 2$ times increase in the flux normalization. There is also weak evidence for spectral hardening $(\Delta \Gamma=0.16)$, however the change in spectral index is consistent within statistical errors.

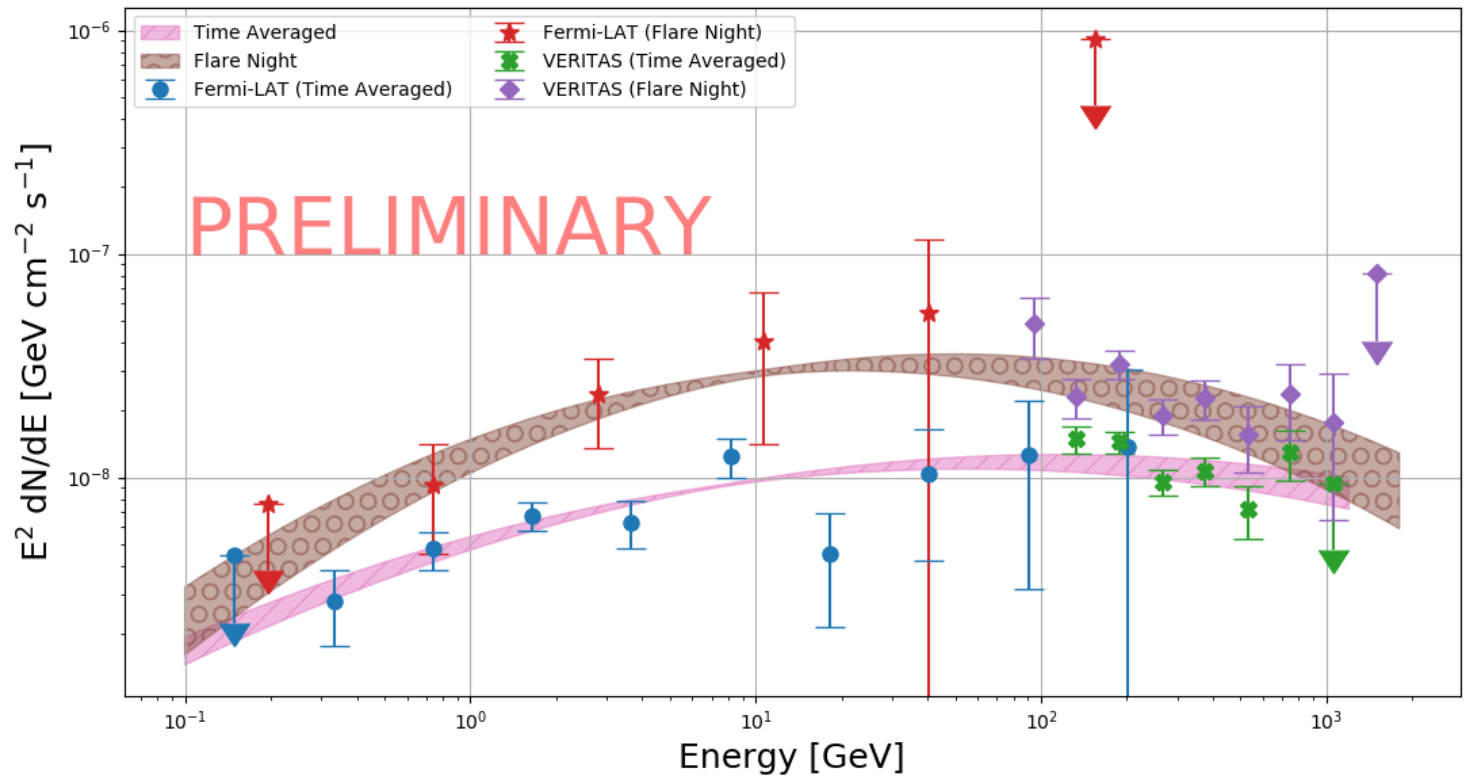

Figure 3: Gamma-ray energy spectrum for 1ES 1218+304 for the time-averaged data set and the night of the "flare". The blue circles and red stars denote the time-averaged and flare-night Fermi-LAT data, respectively. The green crosses and purple diamonds denote the time-averaged and flare-night VERITAS data, respectively. The pink crossed and brown dotted fills denote the best-fit log-parabola models for the timeaveraged and flare night data, respectively. The data is deabsorbed assuming a [16] EBL model. See text for full details.

The Fermi-LAT energy spectra were obtained for both the time-averaged and the flare-night data. In taking data for the flare night, all observations taken within \pm 0.5 days of the VERITAS observations were considered. This ensures sufficient statistics are available when applying the fit. The flare-night LAT data set results in a significant detection, with a TS of 74, assuming a power-law model. The results of the time-averaged and flare-night spectral fits are reported in Table 1 and are shown in Figure 3 as blue circles and red stars, respectively. 95\% C.L. upper limits are shown for energy bins with $T S<9$, corresponding to $\lesssim 3 \sigma$ detection. Comparing the best-fit spectral index for two epoch, one sees evidence of spectral hardening $\Delta \Gamma=0.32$, however this is also weak evidence as the change in spectral index is a $<2 \sigma$ deviation between the time-averaged and flare-night fits.

To characterize the high-energy SED, a joint fit is applied to the Fermi-LAT and VERITAS data 


\begin{tabular}{|c|c|c|c|c|c|}
\hline Dataset & $\begin{array}{c}\mathrm{N} \\
\left(\mathrm{cm}^{-2} \mathrm{~s}^{-1} \mathrm{TeV}^{-1}\right) \\
\text { (2) }\end{array}$ & (3) & (4) & $\begin{array}{c}E_{0} \\
(\mathrm{TeV}) \\
(5)\end{array}$ & $\begin{array}{c}\chi^{2} / N D F \\
(6)\end{array}$ \\
\hline (Time Averaged) & & & & & \\
\hline Fermi-LAT & $(3.44 \pm 0.36) \times 10^{-7}$ & $-1.74 \pm 0.06$ & N/A & $4.57 \times 10^{-3}$ & N/A \\
\hline VERITAS & $(2.42 \pm 0.15) \times 10^{-10}$ & $-3.25 \pm 0.11$ & N/A & $2.00 \times 10^{-1}$ & $13.1 / 5(2.6)$ \\
\hline Combined & $(8.19 \pm 0.84) \times 10^{-8}$ & $-1.83 \pm 0.03$ & $-0.10 \pm 0.03$ & $1.10 \times 10^{-2}$ & $20.4 / 12(1.7)$ \\
\hline (Flare Night) & & & & & \\
\hline Fermi-LAT & $(1.16 \pm 0.35) \times 10^{-6}$ & $-1.42 \pm 0.17$ & N/A & $4.57 \times 10^{-3}$ & N/A \\
\hline VERITAS & $(4.71 \pm 0.37) \times 10^{-10}$ & $-3.09 \pm 0.13$ & N/A & $2.00 \times 10^{-1}$ & $9.4 / 7(1.3)$ \\
\hline Combined & $(1.69 \pm 0.41) \times 10^{-7}$ & $-1.86 \pm 0.07$ & $-0.18 \pm 0.07$ & $1.34 \times 10^{-2}$ & $8.4 / 9(0.9)$ \\
\hline
\end{tabular}

Table 1: Fit details for the the different datasets considered. Column (1) shows the data set, Column (2) shows the Normalization at refernce energy given by Column (5). Columns (3) and (4) show the spectral index and curvature parameter where applicable. Column (6) shows the $\chi^{2}$ per degree of freedom for the fit. See text for more details about the fitting procedure.

sets. The data sets were first deabsorbed for EBL absorption assuming a [16] EBL model. Using the Sherpa python package [17], the spectral points for both the Fermi-LAT and VERITAS data were fit with a power-law and log-parabola model. In applying these fits, the upper limits were excluded from the fitting procedure. In both the case of the time-averaged and the flare-night data sets, were best-fit by the log-parabola model. The details of these fits are shown in Table 1 and the 1- $\sigma$ confidence interval on the best fits are shown as shaded regions in Figure 3. Comparing the best-fit log-parabola model of the time-averaged and flare-night data set show that the spectral index and curvature parameters remain consistent between the different epoch, with flux normalization varying by a factor of $\sim 2$. This is consistent with the observed change in flux in the VHE regime.

\section{Conclusions}

This work represents details of the 2018-2019 monitoring campaign of 1ES 1218+304 . During this campaign, enhanced MWL activity was observed from optical to gamma-ray energies. The analysis of the gamma-ray data during this period reveal a nightly-scale variability, allowing for high-statistics spectra for the time-averaged and flare-night states. The HE SEDs are obtained in the $100 \mathrm{MeV}$ to $>1 \mathrm{TeV}$ energy range, revealing remarkable hard VHE spectra and IC-peaks in the 10s-100s of Gev range.

VERITAS will continue to monitor 1ES 1218+304 and other VHE blazars, as well as initate ToO observations on promising targets triggered by MWL activity. In the event of enhanced VHE emission, VERITAS will alert multiwavelength partners and the wider astronomy community via astronomers telegrams.

\section{Acknowledgement}

This research is supported by grants from the U.S. Department of Energy Office of Science, the U.S. National Science Foundation and the Smithsonian Institution, and by NSERC in Canada. This research used resources provided by the Open Science Grid, which is supported by the National 
Science Foundation and the U.S. Department of Energy's Office of Science, and resources of the National Energy Research Scientific Computing Center (NERSC), a U.S. Department of Energy Office of Science User Facility operated under Contract No. DE-AC02-05CH11231. We acknowledge the excellent work of the technical support staff at the Fred Lawrence Whipple Observatory and at the collaborating institutions in the construction and operation of the instrument.

\section{References}

[1] J. Albert et al., Discovery of Very High Energy Gamma Rays from 1ES 1218+30.4, ApJL, 642, L119, (2006).

[2] V. A. Acciari et al., VERITAS Observations of the BL Lac Object 1ES 1218+304, ApJ, 695, 1370, (2009).

[3] R. Mukherjee et al., VERITAS Detection of an Exceptional VHE Gamma-Ray Flare from the Blazar IES 1218+304, The Astronomer's Telegram, 12360, (2019).

[4] R. Mirzoyan, MAGIC detects an unprecedented activity from the blazar 1ES 1218+304 at very high energy gamma rays, The Astronomer's Telegram, 12365, (2019).

[5] V. F. Bonneli et al., The Highest Historical X-ray Brightness State in HBL Source 1ES 1218+304, The Astronomer's Telegram, 12354, (2019).

[6] N. Park, Performance of the VERITAS experiment, in proceedings of 34th ICRC, POS (ICRC2015) 771 (2015).

[7] M. Krause et al., Improved $\gamma /$ hadron separation for the detection of faint $\gamma$-ray sources using boosted decision trees, ApP, 89, 1 (2017).

[8] T.-P. Li, and Y.-Q. Ma, Analysis methods for results in gamma-ray astronomy, ApJ, 272, 317, (1983).

[9] W. B. Atwood et al., The Large Area Telescope on the Fermi Gamma-Ray Space Telescope Mission, , 697, 1071 (2009).

[10] M. Wood, Fermipy: An open-source Python package for analysis of Fermi-LAT Data, in proceedings of 35th ICRC, POS ( ICRC2017) 824 (2017).

[11] F. Acero et al., Fermi Large Area Telescope Third Source Catalog, ApJS, 218, 23 (2015).

[12] J. D Scargle et al., Studies in Astronomical Time Series Analysis. VI. Bayesian Block Representations, ApJ, 764, 167, (2013).

[13] Astropy Collaboration, The Astropy Project: Building an Open-science Project and Status of the v2.0 Core Package, Astronomical Journal, 156, 123, (2018).

[14] F. Piron et al. 2001, Temporal and spectral gamma-ray properties of Mkn 421 above $250 \mathrm{GeV}$ from CAT observations between 1996 and 2000, A\&A, 374, 895, (2001).

[15] W. A. Rolke et al., Limits and confidence intervals in the presence of nuisance parameters, NIMPA, 551, 493, (2005).

[16] A. Domínguez et al., Extragalactic background light inferred from AEGIS galaxy-SED-type fractions, MNRAS, 410, 2556, (2011).

[17] P. Freeman et al., Sherpa: a mission-independent data analysis application, Proc. SPIE 4477, Astronomical Data Analysis, (2001). 